\title{
Research on Product Design based on IP of Tujia Culture
}

\author{
Peng Hong \\ Wuhan University of Science and Technology \\ Wuhan, Hubei, China
}

\author{
Tao Weitong* \\ Wuhan University of Science and Technology \\ Wuhan, Hubei, China
}

\author{
Liu Yunxian \\ Wuhan University of Science and Technology \\ Wuhan, Hubei, China
}

\begin{abstract}
Tujia ethnic nationality mainly distributes in Wuling Mountains. The ancestors of Tujia people were called "Wuling Barbarians" or "Wuxi Barbarians". The honest and straightforward folk custom of Tujia nationality has created the intuitive, plain, clean and neat artistic characteristics of Xilankapu. Its cultural intellectual property is highly recognized and has a strong "modern design gene". This study takes Tujia brocade as the research object, analyzes the innovative design concept, improves the design from Kapp's pattern and color, adopts various design techniques and traditional cultural connotations, so as to make the products are stamped with characteristics of the times and explore new ways of national culture innovation.
\end{abstract}

Keyword-Tujia nationality; Xilankapu; Culture IP; Product design

\section{INTRODUCTION}

As a traditional handicraft art of the Tujia nationality, Tujia brocade has distinctive national features, but it has encountered problems during industrialization: on the one hand, Xilankapu (Tujia brocade) is rarely known to people other than Tujia people and academic researchers. The promotion strength is not enough; on the other hand, traditional craftsmen are getting older and older, and there is no successor; because the hand-made brocade is expensive, it attracts little attention; and the woven Tujia brocade is not innovative in designing, and the derivative products are not modern enough. At present, Xilankapu product design is mainly used in wall hanging, table flag, throw pillow, bedding, clothing, blanket and other soft decoration. Other designs on Xilankapu basically use traditional patterns and colors, so the "value of fashion" is not fully shown. This research mainly deconstructs and reorganizes the dermatoglyphic pattern of Xilankapu, and innovates the color design to better show its implied meaning, upgrade the commercial value of Xilankapu, make the product fashionable and modern, make the traditional culture IP rejuvenate itself and allow the national art exist forever.

\section{An Overview of Tujia ethnic Culture IP}

In cnki.net, there are 34,593 documents with the key word of "Tujia nationality", including 1,578 ones about Tujia brocade, 222 ones about Xipulanka in Tujia, 866 ones about Tujia music, and 31,333 ones on other themes. Most documents study the architecture, living habit, daily necessity, clothing, mask and others. With the keywords of "Tujia nationality" + "Culture", there are 25,918 documents. And with the keywords of "Tujia nationality" + "Culture" + "Art", there are 8,740 documents. The specific data is detailed in Table 1 below.

TABLE I NUMBER OF DOCUMENTS WITH DifFERENT KEYWORDS IN CNKI.NET

\begin{tabular}{|c|c|c|c|c|c|c|}
\hline Keyword & $\begin{array}{c}\text { Number of } \\
\text { Documents }\end{array}$ & $\begin{array}{c}\text { Tujia } \\
\text { Brocade }\end{array}$ & Xilankapu & Music & Dance & Others \\
\hline $\begin{array}{c}\text { Tujia } \\
\text { Nationality }\end{array}$ & 34593 & 1578 & 222 & 866 & 594 & 31333 \\
\hline $\begin{array}{c}\text { Tujia } \\
\text { Nationality } \\
+ \text { Culture }\end{array}$ & 25918 & 1389 & 209 & 618 & 520 & 23182 \\
\hline $\begin{array}{c}\text { Tujia } \\
\text { Nationality } \\
+ \text { Culture } \\
\text { Art }\end{array}$ & 8740 & 1119 & 169 & 401 & 322 & 6729 \\
\hline
\end{tabular}

From the above table, we can see that Tujia brocade and Xilankapu have always occupied the top position of the culture of Tujia nationality. The number of studies on Tujia brocade has always remained the largest in the fields of research on ethnic group, national culture as well as national culture and art.

At the end of the 20th century, China began to deeply research on the Xilankapu culture. In 1985, Mr. Tian Shunxin's article "History of Tujia Brocade and Discussion on the Color and Organization of Traditional Pattern"(1) mainly studied the historical and artistic characteristics of Xilankapu. Later, more and more scholars participate in the research of Xilankapu, which improves public understanding of Tujia culture. Based on the above researches, it can be inferred that "Xilankapu" can be used as the visual and cultural IP of the Tujia nationality. 
IP (Intellectual Property) means intellectual property. The construction of IP is equivalent to that of culture in a sense, and it is a cultural image with its own characteristics ${ }^{2}$. As the most representative visual IP of Tujia culture, Xilankapu has a profound cultural foundation and serves as a concrete form of cultural carrier to disseminate content related to Tujia culture.

In Tujia dialect, "Xilankapu" is a kind of satin quilt. It is not only a daily necessity for the Tujia people for generations, but also a dowry for Tujia girls. Because of its beauty and auspicious meaning, it has been passed down to the present. Its patterns mainly include: patterns based on natural plants and flowers, animals, living utensils and other living equipment, folktales and folk legends, folk customs and characters as well as modern and innovative patterns. It is the representative of Tujia national wisdom, craftsmanship, and an important national art feature of Tujia nationality. It expresses the aesthetic feelings of Tujia people, has unique cultural characteristics and aesthetic value ${ }^{(3)}$ and reflects the rich national life connotation and desire for a good life. The tools for the Tujia brocade are very simple and primitive, but their unique technology, namely the warp, weft and oblique three-line weaving technique ${ }^{4}$, basically follows the ancient traditional brocading method. The brocading pays attention to the color matching and creation of the pattern. The geometric shape is the basic shape and has certain regularity. Many patterns are created by symmetrical pictures which are repeatedly arranged.

With the integration of national cultures, the development of urbanization and industrialization, the Tujia culture has been greatly impacted. Many of hundreds of traditional patterns have been lost. As the country explores national resources and develops national culture, Xilankapu has attracted new attention.

\section{AN INTRODUCTION TO EXISTING TUJIA BROCADED PRODUCTS}

The existing Tujia brocaded products mainly include: bedding (with the cover pattern of local flowers), sachet, clothing, tour bag, sofa cover, cushion, interior decoration, armor, backpack, mobile phone shell, furniture, travel craft and so on; and the Xilankapu pattern is used in package design, logo design, graphic design, apparel design (pattern, shell fabric, color) and other fields. Most Tujia brocade patterns are used in soft decoration design. In other fields, the Tujia brocade pattern is little used.

Tujia brocaded products mainly focus on "people". At the beginning, original single (Xilankapu) material is only used. And now, it is combined various materials. Today`s Tujia brocaded products are colorful. All brocade bags for sales are only the application of Tujia brocade technology. The improvement and innovation on the technology is rare. Through the investigation of Xilankapu series products of "Tujia Mountain Village", "Guaiyaomei" and other Tujia brocade brands in western Hubei and Hunan, we find some problems: the color and the pattern are too traditional; product form is single, homogenization is serious and innovation is lacking; the product is not fashionable enough, and there is a lack of awareness of creating a characteristic brand; there is no different enterprise positioning. If the innovative design and color of the pattern are used ingeniously and the "modern design gene" can be explored and used reasonably, the design will be closer to the modern aesthetic standard. Based on the traditional design, the improved design makes the existing products get rid of complicated form to carry out design innovation to meet the needs of modern life.

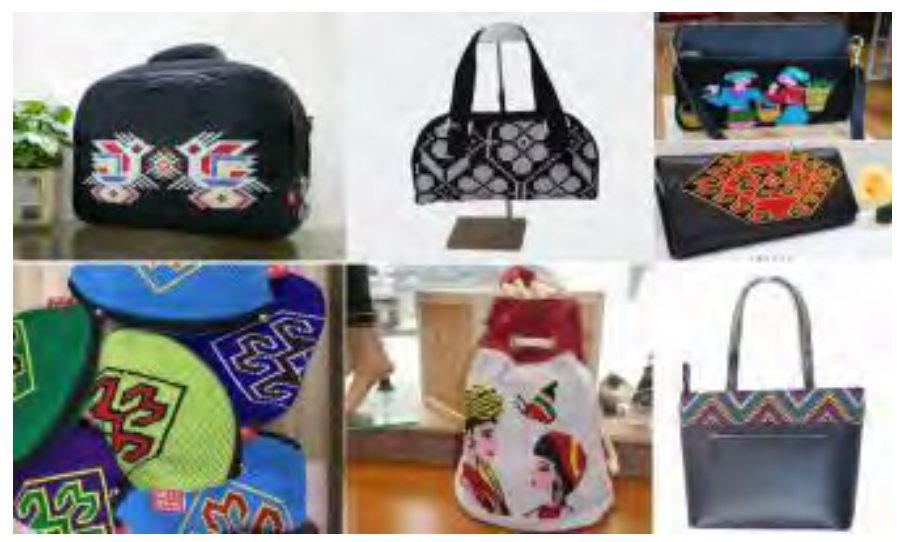

Fig. 1 Existing Designs of Tujia Brocaded Bags

\section{INVESTIGATION AND FEASIBILITY STUDY ON TUJIA BROCADING IN PRODUCT DESIGN}

The Xilankapu pattern has not only the essence of the traditional culture of the Chinese nation, but also its own features of national culture. The research begins with the basic definition, historical origin and research value of Tujia brocade, and refines its art, color, craft and culture characteristics. It also classify the design principles of Xilankapu, application methods and manifestation patterns used in product design. It studies the mutual penetration and union between product design and pattern.

The traditional patterns of Xilankapu are cumbersome and diverse. The straight line is always used ${ }^{(6)}$, which is in line with the simple needs of modern product design. The "modern design gene" in the pattern — a large number of geometric shapes can be used for secondary design. From the aspects of the shape, color and arrangement of the Xilankapu pattern, the pattern can be redesigned by means of reorganization, reconstruction, transplantation and other methods to improve the commercial application space of Xilankapu and make the products fashionable.

The color matching of Tujia brocade largely retains the essential characteristics of color. The most distinctive feature is that contrasting colors can be matched in the same piece without any discord. Generally, dark blue or black is used as the bottom color. And other colors are matched based on it. There are also some rules used in the area of the color block, which make the color layering but not messy. The colors of traditional Tujia brocade patterns range from three or four to more than a dozen.

The Xilankapu pattern of Tujia brocade in the product design pays attention to the area, color matching, white space and other aspects, which is shown in the combination of modern and traditional aesthetics, the emotional echo, and the relationship between products. This research redesigns the pattern through the imitation of the shape and color of the 
Xilankapu pattern and the combination between the modern design and the traditional one, which not only retains the national characteristics but also the fashion sense. In the actual creation, in term of the innovative design or deformation design of the Xilankapu pattern, the selection of auspicious and hilarious spider flower, flowing water flower, single eight-hook pattern and the application of redesigning of the wallet, handbag, bedding, silk, table flag and others focus on the area and distribution of patterns to make pattern and design aesthetics harmonious.

\section{CASE Study}

Tujia culture IP includes pattern gene, color gene, cultural style gene and others. The geometric pattern of Xilankapu is extracted, and the second design is carried out by modern design methods such as folding, rotation, repetition and reconstruction to preserve its cultural style. The simple color gene retains auspicious meaning and carries out modern designing and application of the traditional Xilankapu.

\section{A. Design of Handbag with Pattern of Spider Flower}

Spider flower (see Figure 2). Tujia nationality thinks that the spider flower symbolizes the accumulation of wealth, a heaven-sent fortune, auspiciousness and good luck. The spiders gather together to celebrate the happy events. And the spider flower is used in the design of the handbag to represent that the money is gathering, and good luck will come.

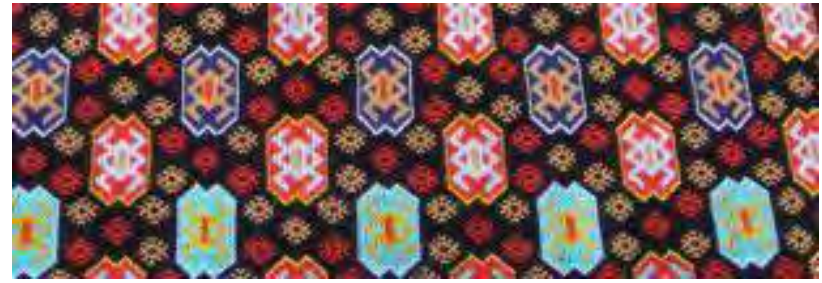

Fig. 2 Traditional Pattern of Spider Flower

The simple geometric shape is the "modern design gene" of traditional spider flower. Changing its fine and complicated form, adopting the methods of deformation, repetition and recombination to make the pattern fashionable, simple but characteristic and in line with modern simple and solemn beauties.

The traditional Xilan Kapu pattern is richer and more colorful. The original colors are simplified. Two colors are alternated, such as red and white. Red represents warmness and auspiciousness, and white represents purity and cleanness, reflecting the simple aesthetic pursuit.

According to the pattern of the spider flower, the auspicious meaning is used on the wallet design to fully play the role of the pattern, so that the wallet carries the characteristics of the Tujia culture IP, trying to make the spider flower present a fresh form.

The spider flower purse and the flowing flower handbag are both created through digital printing, which makes the application of the Xilankapu pattern wider. And the printing material is easier to clean than the traditional hand-woven brocade. The printing material is suitable for the principle of using the handbag. Different visual effects are formed, and the price is more affordable. The production process is also a major problem to be solved in the production of Tujia brocade. The rational use of modern science and technology is also conducive to market development and brand expansion ${ }^{(7)}$ (see Figure 3).

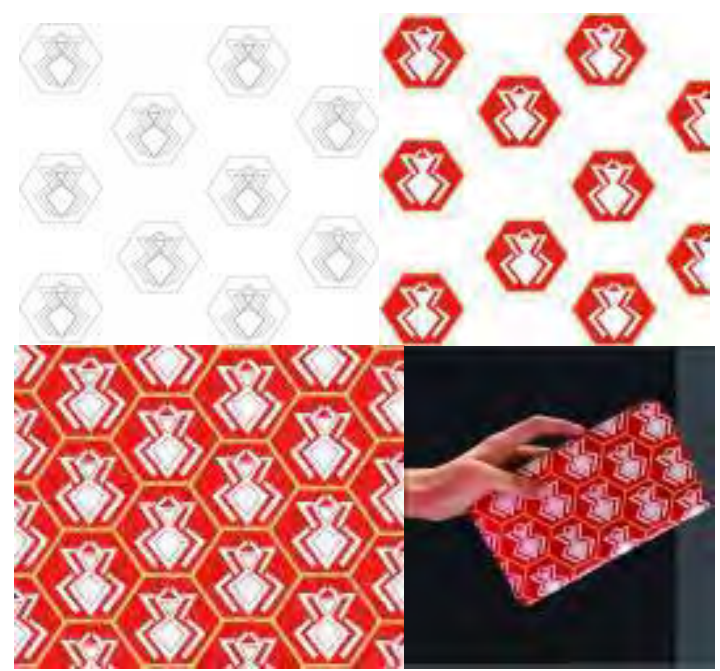

Fig. 3 Design Scheme of Spider Flower Designed by Tao Weitong

\section{B. Design and Application of Single Eight-Hook Pattern Based on Tujia Brocade and Xilankapu}

With symmetrical hook patterns with same size, Single eight-hook flower has a profound meaning. There are four sets of geometric hooks in a single eight-hook flower. The upper and lower sets of hooks represent newly married couples, family members and ancestors. The left and right sets of hooks represent the world. The meaning of the hook pattern is enjoying a happy life, and everything going well. It expresses the desire of the Tujia people for a happy and beautiful life. This beautiful wish is woven into the single eight-hook flower and passed on to next generations ${ }^{8}$ (see Figure 4).

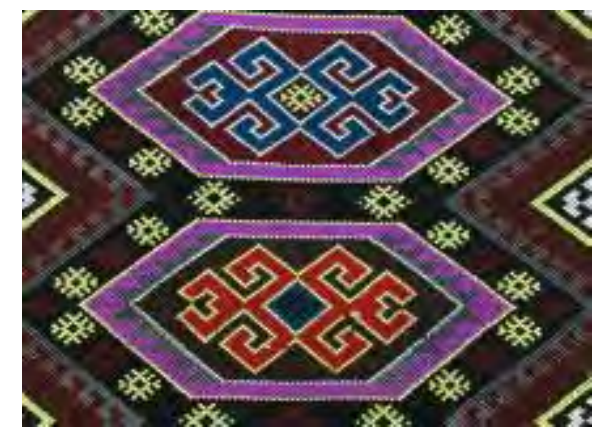

Fig. 4 Traditional Single Eight-Hook Pattern

The design of the eight-hook flower gets rid of the complexity of the traditional single eight hook pattern. The "modern design gene" _ simple geometric figures, that is the hook patterns are re-arranged and combined. The patterns are distributed evenly and repeated without limit. The combinations of thick and fine lines as well as single eight-hook flowers and “万”-shaped patterns make the whole pattern richer and more harmonious (see Figure 5). 
The traditional Tujia brocade pattern is colorful $^{9}$.Red symbolizes auspiciousness. A combination of red and white is always used. Pay attention to the relationship between pattern distribution and overall white space and coordinate the color proportion. And the advantages of traditional Tujia brocade pattern color using contrasting colors shall be learned to unify the spirit and aesthetics to make the product and the user echo in emotion.

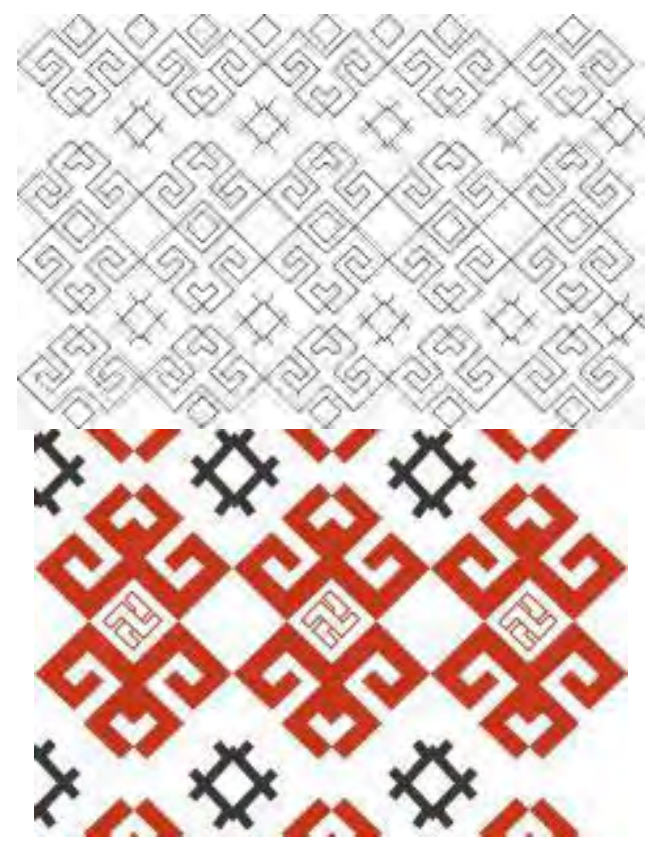

Fig. 5 Scheme of Combination of Single Eight-Hook Pattern and “万”-Shaped Pattern

Apply the single eight-hook pattern to silk, bed sheet, quilt, pillow, and storage bag (see Figures 6 and 7).

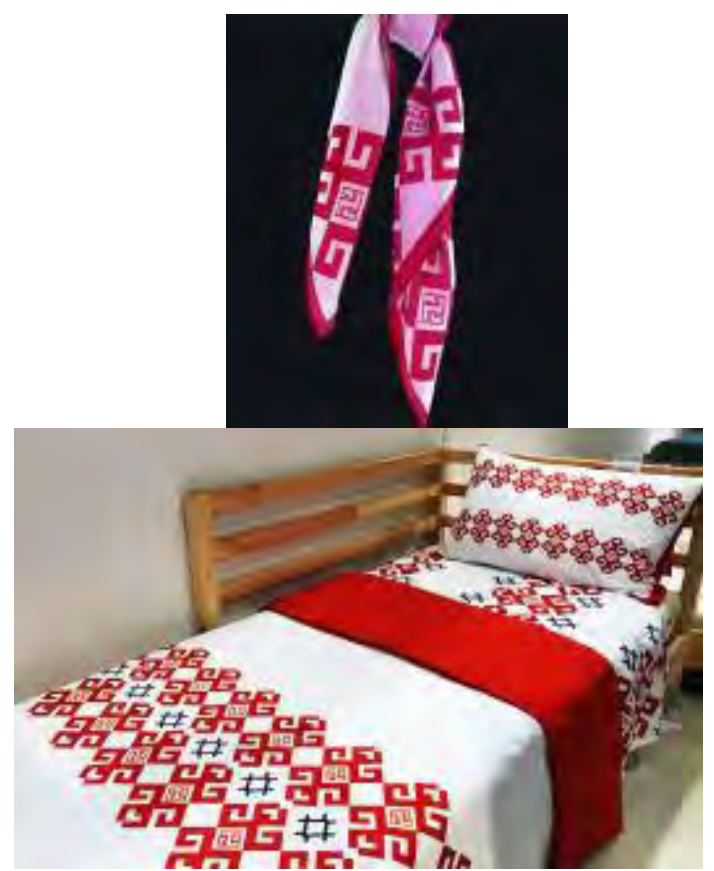

Fig. 6 Design Sketch of Products with Single Eight-Hook Pattern Designed by Tao Weitong

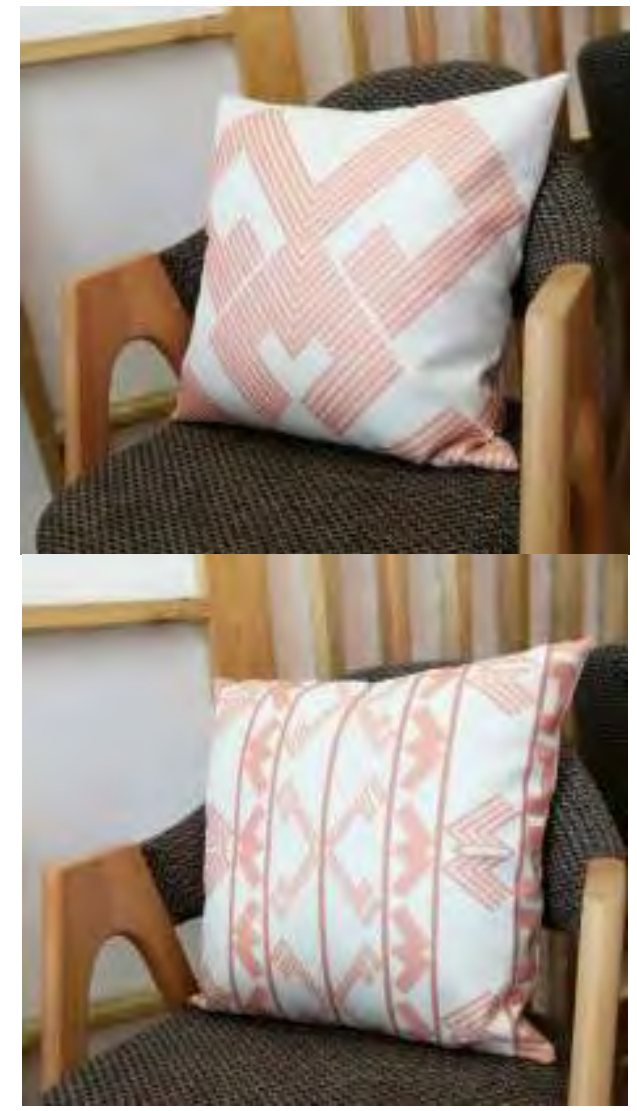

Fig. 7 Finished Products with Single Eight-Hook Pattern and Examples of the Pattern Designed by Shang Qianli

In Figure 7, pink is mainly used in the products. The product positioning is that it is used in young women's room. It emphasizes soft temperament, so the color should not be too much. Other color scheme is the simple combination of black and white, which is more visually impactful and suitable for use in eye-catching places. It is designed for use in floor mat, storage bag and others (Figure 8).The re-combined pattern of Xilankapu has some exotic temperament (Islamic style), and the innovative design based on modern fashion trends also enriches the application of Xilankapu. 


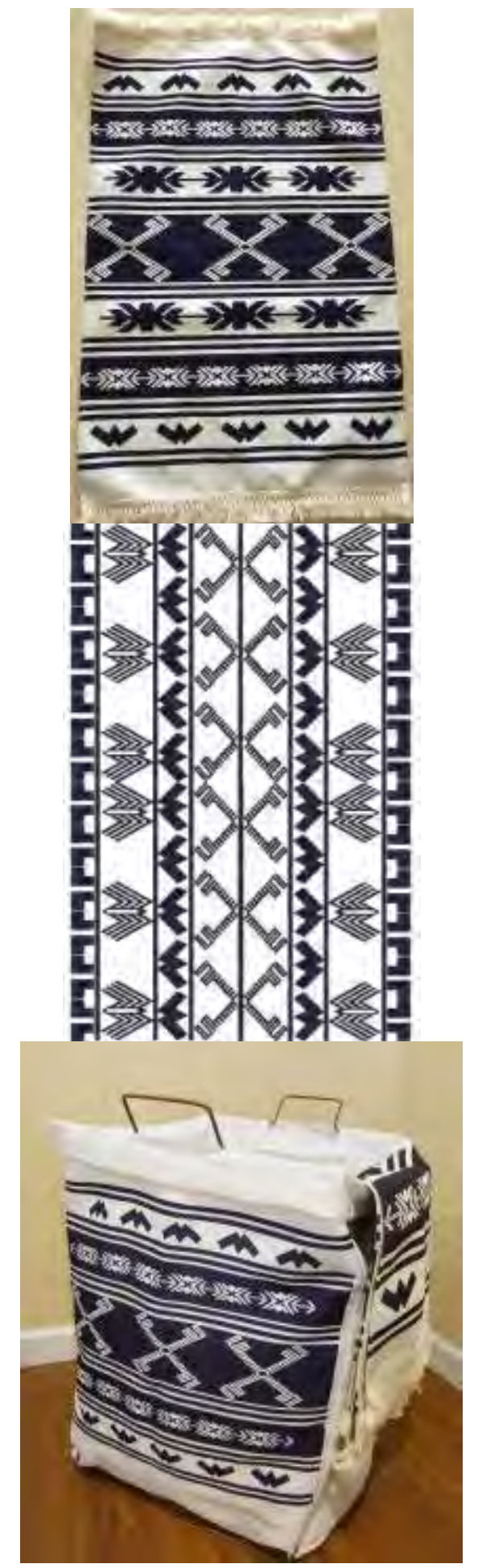

Fig. 8 Single Eight-Hook Pattern Design Color Scheme and Application in Carpet Designed by Shang Qianli

\section{Reconstruction of Flowing Water Flower Pattern Based on “万” Character and Product Design}

The “万”-shaped flowing water flower is an auspicious pattern with Tujia style. It implies the auspiciousness, happiness, the desire for a bright future as well as well-being. Tujia people believe that it can bring peace and good luck to people. It is not only a reflection of faith, but also an expression of traditional auspicious pattern ${ }^{(10)}$ (see Figure 9)

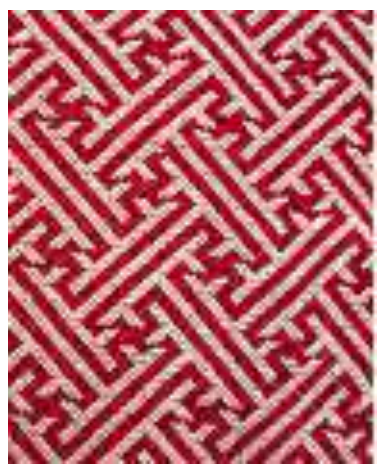

Fig. 9 Traditional “万”-Shaped Flowing Water Flower

The traditional “万”-shaped flowing water flower is broken up and reconstructed to form a hierarchical structure between the figures, give people a dynamic visual effect, avoid the visual fatigue of the traditional arrangement, so that the arrangement of the patterns can be more spatial. Relatively free and random composition can generate a strong rhythmic beauty and make the composition more harmonious to meet modern aesthetic feeling. It seems that the flowing water flowers are disorderly and irregular. Actually, it forms a relaxed and casual artistic style.

The flowing water flower is redesigned. The arrangement and color use of the flowing water flower is refreshed. The gradual change of the contrasting colors of red and white is used to make the color more fashionable. And the contrast between red and white makes the color matching more free and romantic.

Through the traditional Tujia brocade, the flowing water flower-patterned table flag is expressed in the form of plain weave by the weaving method of weft breaking. The frequency of using the traditional handicraft as a production method in the modern market is also increasing. Combined with the innovative design of nostalgic style, the product can be distinctive. And the cultural added value of products is enhanced, a cultural and creative design with profound national culture is formed.

The art style, meaning, and modern design gene of the flowing water flower-pattern shall be focused. And exploration and research shall be carried out by combining water flow and product design. Applying the layered flowing water-flower pattern to the table flag design can make the table flag which is longer than other products have a sense of connection. The napkins placed on the table and the table flag echo with each other, giving the eaters a sense of happiness and increasing their appetite. The color matching of the flowing water flower 
can be simply used in the handbag designing to match with the clothing, which has a unique sense of fashion (see Figure 10).

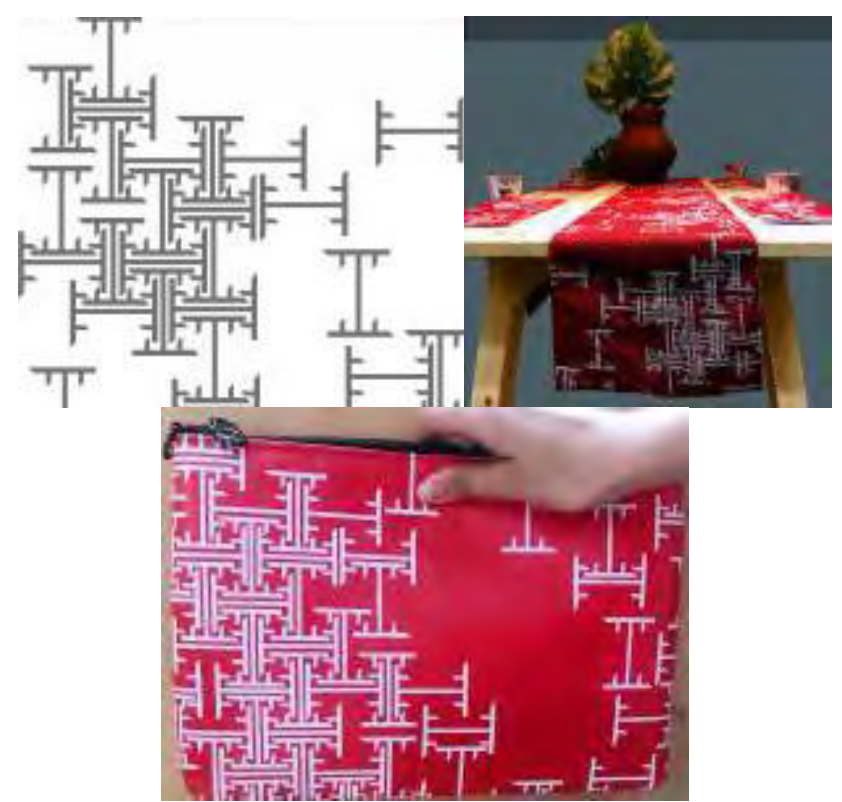

Fig. 10 Design and Application of Flowing Water Flower in Table Flag and Handbag Designed by Tao Weitong

\section{CONCLUSION}

Xilankapu is of high historical research value and significance. It is a national culture IP with a good "modern design gene". The Tujia culture IP is researched and skillfully applied in the product design. The geometric shapes and color elements of Xilankapu are broken up, reconstructed and applied to product design. The new product is certain decorative and have good meanings. It can satisfy the substances and spirit requirement of consumers, and solve the problems of the Tujia brocade, such as single product form and serious homogenization. Dig deeper and combine with the carrier to find new value points. The focus of this research is the innovation and fashion of the pattern. The follow-up groups will continue to explore the national culture IP and create products with unique national characteristics.

\section{ACKNOWLEDGMENT}

Fund Project: This article is the staged achievement of the art project of 2017 National Social Science Fund: "Research on the Development of Cultural and Creative Products Based on Museum Rating and Evaluation Standards" (No. 17BH160).

\section{REFERENCES}

[1] Zhou Chenghua. "On the Abstract Composition of Xilankapu" [J]. Zhuang Shi, (05):64.

[2] Sun Jiaqi, Wang Yating, Tang Hongmei. "Research on Jinggangshan Red Tourism Product Marketing Based on Cultural IP'[J]. CO-Oerativeconomy \& Science, 2018(09): 134-135.

[3] Zhou Liya, Gu Yi. "Explore the Implications of Tujia Brocade Art"[J]. Zhuang Shi, 2004(04): 88-89.

[4] Liu Yizhong. "The National Characteristics of Tujia Brocade Art"[J]. Zhuang Shi, 2006(10): 37-38.

[5] Lu Yimei, Lu Qian. "The Aesthetic Abstraction of Tujia Brocade from the Perspective of Pattern and Color"'J]. Zhuang Shi, 2009(08): 116-117.

[6] Wang Wei. "Research on Redesign of Residual Materials Based on Patchwork Theory-Take Tujia Brocade as an Example"[J]. Zhuang Shi, 2017(09): 140-141.

[7] Liu Guanbin. "Research on the Evolution of Tujia Brocade Hook Pattern"[J]. Zhuang Shi, 2017(11): 124-125.

[8] Su Xiao. "Research on the Application of Tujia Brocade Pattern in Soft Decoration Design" [J]. Yi Hai, 2017 (02): 97-98.

[9] Meng Jun. "Analysis on the Xilankapu Pattern and Color Rheology of Tujia Brocade" [J]. Lantai World, 2017.

[10] Meng Jun. Research on the Pattern Elements of Xilankapu and Its Implications [J]. Mei Yu Shi Dai (I), 2017. 